\title{
Anisotropic transport behavior in ultrathin epitaxial Fe films on vicinal oxide substrates
}

\author{
Xuesong Jin, ${ }^{\text {a) }}$ Ciaran McEvoy, and I. V. Shvets \\ Science Foundation Ireland (SFI) Nanoscience Laboratory, Physics Department, Trinity College Dublin, \\ Dublin 2, Ireland
}

(Presented on 9 November 2004; published online 13 May 2005)

\begin{abstract}
Step-induced anisotropy of electron transport in ultrathin Fe film was investigated. The Fe films (2 and $10 \mathrm{~nm}$ ) were deposited on vicinal $\mathrm{MgO}$ (100) substrate using molecular-beam epitaxy. It is found that the films with a thickness of 10 and $2 \mathrm{~nm}$ are continuous and discontinuous, respectively, which was determined from their resistivity values, the temperature dependency of the resistivity and the $V-I$ curve. The enhanced magnetoresistance in the continuous and the discontinuous films was observed when the current flows parallel and perpendicular to the miscut direction, respectively. We suggest that the atomic steps in the continuous films nucleate additional domain walls acting as scattering centers and the extra scattering was introduced for the current perpendicular to the step edges. The mechanism of the influence of the atomic steps on the electron-transport properties is different in the continuous and discontinuous films. We further suggest that in the discontinuous films, the atomic steps result in the anisotropic growth of the islands and the anisotropy in the transport behavior as a consequence. () 2005 American Institute of Physics.
\end{abstract}

[DOI: $10.1063 / 1.1847915]$

\section{INTRODUCTION}

Vicinal low-index surfaces provide interesting substrates for film growth because of the existence of regular arrays of atomic steps. The effect of atomic steps on the electrontransport, magnetic, and optical properties of superconducting films, semiconductor, and metal films has been extensively investigated. A step edge pinning was observed in $\mathrm{Bi}-$ 2201 superconducting films. ${ }^{1}$ This phenomenon could be employed to enhance the critical magnetic field of superconducting films. A large anisotropy in the electron conductivity has been found on the GaAs substrate. ${ }^{2,3}$ Several theoretical works were carried out on the topic. ${ }^{4,5}$ In this paper, we study the influence of the surface miscut from a low-index plane of the $\mathrm{MgO}(100)$ substrate on the electron-transport properties of ultrathin epitaxial continuous and discontinuous Fe films. The role of the surface miscut on the transport properties of the continuous and discontinuous films was investigated. The rationale for the study is to create the array of the atomic steps preferentially aligned perpendicular to the miscut direction. Although the presence of atomic steps on a miscut substrate is clearly expected we do not directly demonstrate their presence in the study. The body of the experimental results presented can be readily explained by the presence of the atomic steps directed, as expected, perpendicular to the miscut direction.

\section{EXPERIMENT}

The 2- and 10-nm-thick Fe films, with cross structures, were grown on single-crystal vicinal $\mathrm{MgO}$ (100) substrates with a size of $10 \times 10 \mathrm{~mm}^{2}$ by molecular-beam epitaxy (MBE). The cross structure was fabricated using a shadow

${ }^{a)}$ Electronic mail: xuesongj@tcd.ie mask. One of the arms of the cross structure was aligned along the miscut direction and the other perpendicular to it. The miscut angle of the $\mathrm{MgO}$ substrate was $2.86 \pm 0.01^{\circ}$ along the (001) direction which was determined using highresolution $x$-ray diffractometer. The base pressure in the growth chamber was lower than $5 \times 10^{-10}$ Torr. The $\mathrm{MgO}$ substrates were annealed in situ in an oxygen and oxygen plasma environment prior to deposition. ${ }^{6}$ A $25-\mathrm{nm} \mathrm{MgO}$ cap layer was deposited on top of the Fe film to prevent oxidation. The substrate temperature during $\mathrm{Fe}$ deposition was $200{ }^{\circ} \mathrm{C}$ and the deposition rate was $0.1 \AA / \mathrm{s}$. In situ reflection high-energy electron diffraction (RHEED) was employed to monitor the growth of the films. The transport and magnetotransport properties of the Fe cross structures were investigated using the four-probe method. The magnetoresistance (MR) is defined as $[R(H)-R(0)] / R(0)$, where $R(H)$ and $R(0)$ is the resistivity with and without field. A switching system was used to perform almost simultaneous measurement of the resistances of two arms of the cross structure, along and perpendicular to the miscut direction, thus ensuring that measurements were directly comparable. An external magnetic field was applied in the film plane and parallel to the applied current.

\section{RESULTS AND DISCUSSION}

RHEED patterns were used to monitor the quality of the vicinal substrates and the epitaxial growth of the Fe structure. Figure 1(a) is the RHEED pattern of a cleaned vicinal $\mathrm{MgO}$ (100) substrate. The diffraction pattern displays the vertical lattice rods and sharp horizontal Kikuchi lines. Figure 1(b) shows a plane $\mathrm{MgO}$ (100) substrate deliberately tilted at $\sim 3^{\circ}$ with respect to the electron beam, which also shows horizontal Kikuchi lines, though not as sharp. From this it was determined that the number of inelastically scat- 
(a)

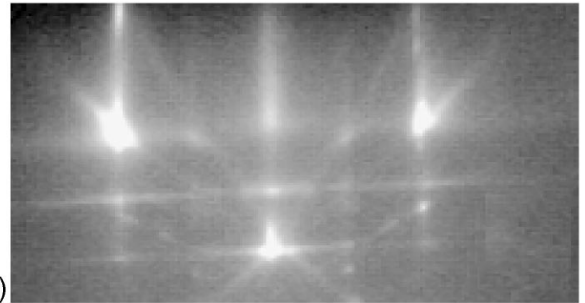

(b)

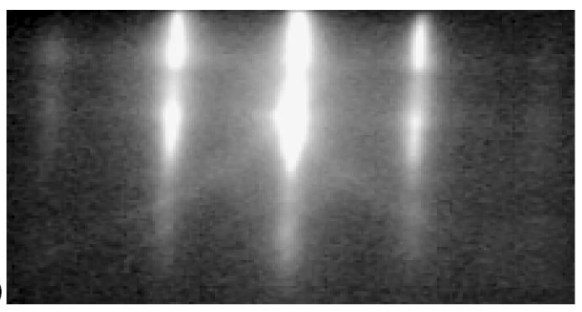

(c)

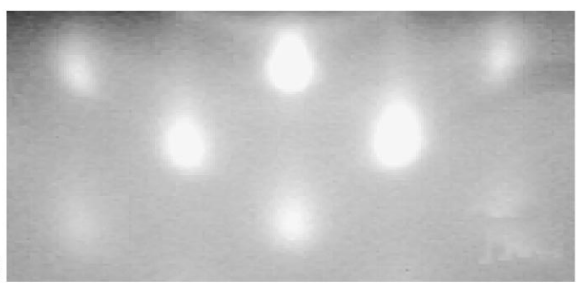

FIG. 1. The RHEED patterns of (a) vicinal $\mathrm{MgO}$ (100) substrate with a miscut angle of $3^{\circ}$, (b) a nonmiscut $\mathrm{MgO}$ (100) substrate which is tilt mounted at about $3^{\circ}$ with respect to the electron beam, and (c) after growth of 10 -nm-thick Fe film on vicinal substrate. All the RHEED patterns are measured along $\langle 110\rangle$ azimuth. The electron energy is $20 \mathrm{KeV}$.

tered electrons was greater for the vicinal substrate, as a result of the surface roughness arising from the atomic steps, thus giving rise to the sharp Kikuchi pattern observed. Figure 1 (c) shows the RHEED pattern for the 10-nm Fe film grown on the vicinal substrate. Epitaxial growth of the Fe film was observed. The epitaxial growth relationship between $\mathrm{Fe}$ and $\mathrm{MgO}$ is known as $\mathrm{Fe}(001) / / \mathrm{MgO}(001)$ and $\mathrm{Fe}\langle 110\rangle / / \mathrm{MgO}\langle 100\rangle .^{7}$ Therefore, each arm of the Fe cross structure was along the $\mathrm{Fe}\langle 110\rangle$ direction thus removing any crystal anisotropic effects.

The resistivity as a function of temperature for the 10and 2-nm-thick films and the current parallel and perpendicular to the expected step edges $\left(\rho_{\|}\right.$and $\left.\rho_{\perp}\right)$ are shown in Figs. 2 and 3, respectively. The 10 -nm-thick film shows a

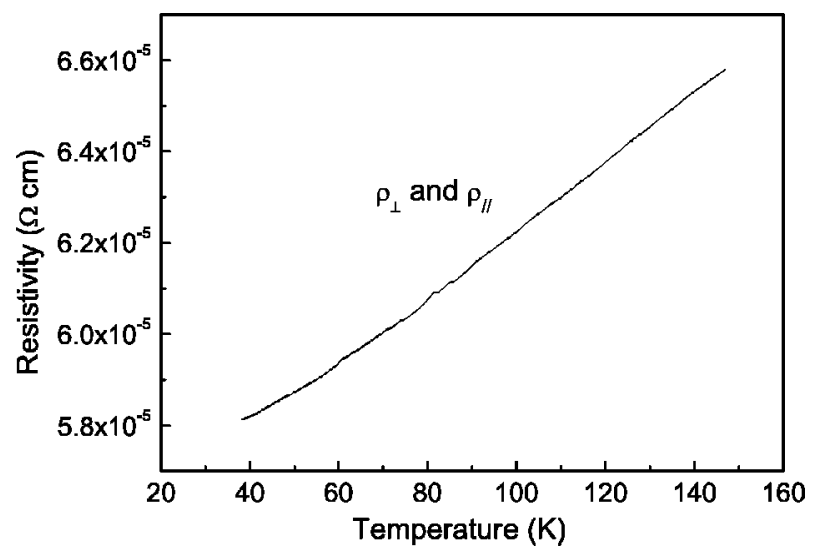

FIG. 2. The resistivity of the 10-nm-thick Fe film as a function of temperature.

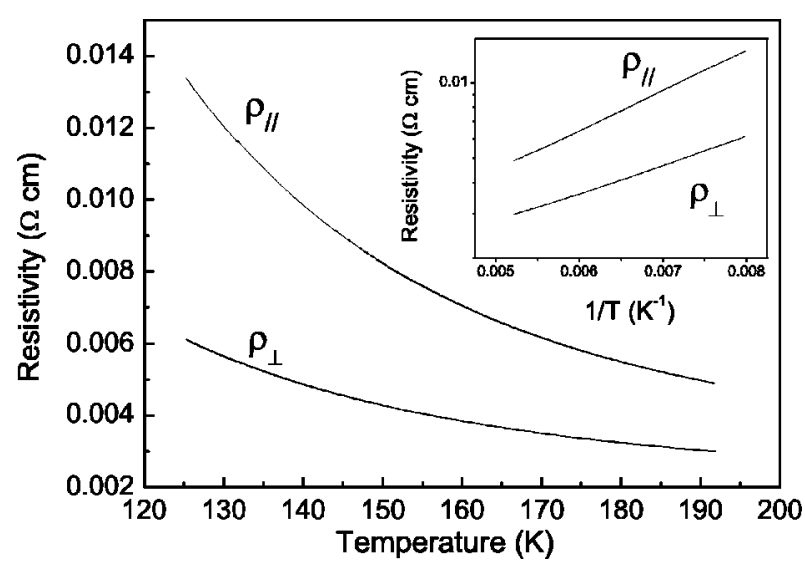

FIG. 3. The temperature dependence of the resistivity along $\left(\rho_{\|}\right)$and perpendicular to the expected step edges $\left(\rho_{\perp}\right)$ for the 2-nm-thick Fe film. The inset shows that the resistivity of film obeys the formula $\ln (\rho) \propto T^{-1}$.

metallic behavior with its resistivity decreasing with decreasing temperature. The $\rho_{\|}$and $\rho_{\perp}$ dependencies for the 10-nm film are identical within the range of experimental errors. A semiconductor behavior, i.e., resistivity of the film increasing with decreasing temperature, was observed for the 2-nmthick film. This, along with much greater values of resistivity for the 2-nm film, reveals its discontinuous nature. In the inset of Fig. 3, it is shown that the resistivity obeys the formula $\ln (\rho) \propto T^{-1}$ that is considered typical for granular metal/insulator systems having a narrow size distribution of metallic granules. ${ }^{8}$ The $V-I$ curve for the 2-nm-thick film shows clear nonlinearity which is consistent with the tunneling between $\mathrm{Fe}$ granules (not shown in this paper). The temperature dependences of MR for 10- and 2-nm-thick films at 2-T field when the current is parallel and perpendicular to the expected step edges $\left(\mathrm{MR}_{\|}\right.$and $\left.\mathrm{MR}_{\perp}\right)$ are shown in Figs. 4(a) and 4(b), respectively. An anisotropy of MR was observed in the films. The sign of MR is different for the two films: it is positive for the 10-nm film and negative for the 2-nm film.

For the $10-\mathrm{nm}$ Fe film, the value of $\mathrm{MR}_{\|}$is smaller than that of $\mathrm{MR}_{\perp}$ in the entire range of measured temperatures. At a temperature of $150 \mathrm{~K}$ there is greater than $40 \%$ enhancement of $\mathrm{MR}_{\perp}$ compared to $\mathrm{MR}_{\|}$. We explain the enhancement in the value of $\mathrm{MR}_{\perp}$ compared to $\mathrm{MR}_{\|}$as follows. The step edges on the surface, being defects in the epitaxial system $\mathrm{Fe} / \mathrm{MgO}(100)$, act as nucleation sites for the additional domain walls in the Fe film. Therefore, a greater number of domain walls are aligned along the step edges rather than perpendicular to them. As a result, electrons moving along the miscut direction undergo a greater number of spin scattering events at the domain walls, resulting in a greater magnetoresistance.

In the case of the 2-nm film, a significant anisotropy in $\rho_{\|}$and $\rho_{\perp}$ was observed. We suggest that the difference is due to the discontinuous structure of the film rather than a result of additional scattering at step edges. In the discontinuous film, the dominant conduction mechanism is the electron hopping between the islands. The thermal activation energy values obtained by fitting the $R$ - $T$ curve are 32.9 and $28.1 \mathrm{meV}$ when the current is along and perpendicular to the expected step edge, respectively. The anisotropy in thermal 


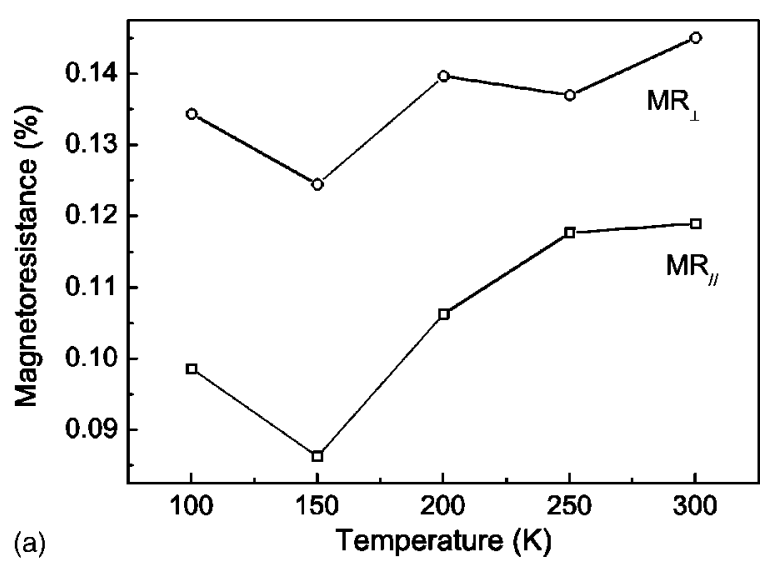

(a)

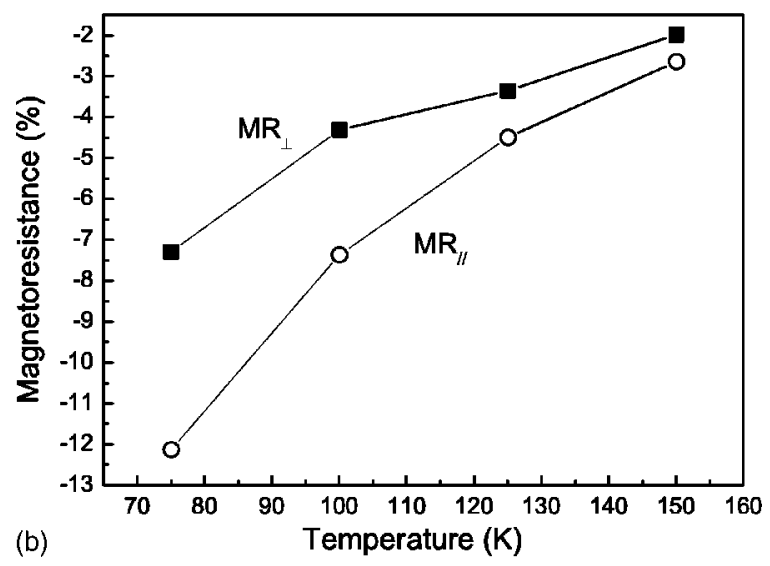

FIG. 4. The temperature dependence of the magnetoresistance along $\left(\mathrm{MR}_{\|}\right)$ and perpendicular to the expected step edges $\left(\mathrm{MR}_{\perp}\right)$ of the (a) 10- and (b) 2-nm-thick Fe films. MR is measured in the field of $2 \mathrm{~T}$.

activation energy implies the existence of an anisotropic tunneling barrier thickness or height along different directions. The barrier height is usually taken as half the band gap of the barrier material, i.e., $\mathrm{MgO}$, in this study. Chainlike scattering potential localized at the steps could create the anisotropy in the resistivity. However, this effect will generate a greater resistivity normal to the expected step. ${ }^{9}$ This is in contrast with our observation where $\rho_{\perp}<\rho_{\|}$. Thus, the anisotropy in the barrier height could not explain the experimental results. We therefore concluded that the anisotropic tunneling barrier thickness is responsible for the observed anisotropy in the resistivity. The barrier thickness is smaller in the direction perpendicular to the expected step edges. The MR results also suggest the existence of an anisotropic barrier thickness. As shown in Fig. $4(\mathrm{~b}), \mathrm{MR}_{\|}$is greater than $\mathrm{MR}_{\perp}$. This is because of the stronger magnetic correlation between the islands along the direction perpendicular to the step edges as a consequence of the smaller barrier thickness along this direction. The decrease in MR at high temperature is mainly due to the suppression of spin-dependent contribution to the conductance by the increase of the spin-independent hopping channel with increase in temperature. ${ }^{10}$ The anisotropic island shape could result in a different tunneling barrier thickness along different directions. Several experiments reveal that the stable two-dimensional island can have a variety of shapes depending on the direction of the crystal surface. ${ }^{11,12}$ This results from the anisotropy of the adatom mobility that is different for different crystallographic directions on the surface during the film growth. Since the crystallographic directions are identical for both arms in the measurements of $\rho_{\|}$and $\rho_{\perp}$, the anisotropic island shape should not result from the anisotropy in the surface diffusion constant of the adatoms. We conclude that the existence of the atomic step structure results in the anisotropic growth of the islands. We have clearly demonstrated the influence of step edges on the island shape in the model system of $\mathrm{Fe}$ islands grown on vicinal Mo (110) substrate. ${ }^{13,14}$

\section{CONCLUSION}

Anisotropic transport behavior in ultrathin epitaxial $\mathrm{Fe}$ film induced by miscut from a low-index plane was investigated. The films with thicknesses of 10 and $2 \mathrm{~nm}$ are continuous and discontinuous, respectively, which is determined from the temperature dependency of the resistivity of the films, the comparison of their resistivity values and the $V-I$ curve. An enhanced magnetoresistance in the continuous and the discontinuous films was observed when the current is directed parallel and perpendicular to the miscut direction, respectively. Although we did not demonstrate the presence of the atomic terraces on the substrate, their presence is clearly expected with the step edges preferentially aligned perpendicular to the miscut direction. Our experimental results can readily be explained with the model implying the existence of the step edges. The mechanism by which the atomic steps influence the electron-transport properties is different in the continuous and discontinuous films. The atomic steps in the continuous films nucleate additional domain walls acting as spin scattering centers when the current flows perpendicular to the step edges. In the discontinuous films, the atomic steps result in the anisotropic growth of the islands and the anisotropy in the transport behavior as a consequence.

\section{ACKNOWLEDGMENT}

This work was supported by Science Foundation Ireland (SFI) under Contract No. 00/PI.1/C042.

${ }^{1}$ Y. Z. Zhang, R. Deltour, J.-F. de Marneffe, Y. L. Qin, L. Li, Z. X. Zhao, A. G. M. Jansen, and P. Wyder, Phys. Rev. B 61. 8675 (2000).

${ }^{2}$ Y. Tokura, T. Saku, S. Tarucha, and Y. Horikoshi, Phys. Rev. B 46, 15558 (1992).

${ }^{3}$ M. Y. Simmons, A. R. Hamilton, S. J. Stevens, D. A. Ritchie, M. Pepper, and A. Kurobe, Appl. Phys. Lett. 70, 1980 (1997).

${ }^{4}$ P. J. Rous, Phys. Rev. B 61, 8484 (2000).

${ }^{5}$ H. Ishida, Phys. Rev. B 52, 10819 (1995).

${ }^{6}$ X. Jin, C. McEvoy, and I. V. Shvets, J. Magn. Magn. Mater. 128-133, 286 (2005).

${ }^{7}$ J. Dekoster, H. Bemelmans, J. De Wachter, R. Moons, and G. Langouche, Appl. Phys. Lett. 65, 1224 (1994).

${ }^{9}$ Y. Tokura, T. Saku, and Y. Horikoshi, Phys. Rev. B 53, R10528 (1996).

${ }^{10}$ C. H. Shang, J. Nowak, R. Jansen, and J. S. Moodera, Phys. Rev. B 58, R2917 (1998).

${ }^{11}$ D. K. Biegelsen, R. D. Bringans, J. E. Northrup, and L.-E. Swartz, Phys. Rev. Lett. 65, 452 (1990).

${ }^{12}$ T. Fukui, S. Ando, Y. Tokura, and T. Toriyama, Appl. Phys. Lett. 58, 2018 (1994).

${ }^{13}$ S. Murphy, G. Mariotto, N. Berdunov, and I. V. Shvets, Phys. Rev. B 68 , 165419 (2003).

${ }^{14}$ S. Murphy, D. Mac Mathuna, G. Mariotto, and I. V. Shvets, Phys. Rev. B 66, 195417 (2002). 